\title{
Reproductive genetic carrier screening for cystic fibrosis, fragile $X$ syndrome, and spinal muscular atrophy in Australia: outcomes of 12,000 tests
}

\author{
Alison Dalton Archibald, PhD, GDipGenetCouns ${ }^{1,2,3}$, Melanie Jane Smith, BSc(Hons) ${ }^{1,2}$, \\ Trent Burgess, BSc(Hons) ${ }^{1,2,3}$, Katrina Louise Scarff, PhD, MGenCouns ${ }^{1}$, \\ Justine Elliott, BSc(Hons), MGenCouns ${ }^{1}$, Clare Elizabeth Hunt, BSc, GradDipGenetCouns ${ }^{1}$, \\ Caitlin Barns-Jenkins, $\mathrm{BSc}^{1,2}$, Chelsea Holt, BAppSc ${ }^{1,2}$, Karina Sandoval, BMedSc ${ }^{1,2}$, \\ Vanessa Siva Kumar, PhD ${ }^{1,2}$, Lisa Ward, BSc ${ }^{1,2}$, Emily Caroline Allen, BSc, MGenCouns ${ }^{2,3}$, \\ Sarah Valerie Collis, MGenCouns, BBmedSc(Hons) $)^{2,3}$, Shannon Cowie, BSc(Hons) ${ }^{1}$, \\ David Francis, MSc, FFSc (RCPA) 1,2 , Martin B. Delatycki, MBBS, PhD $1,2,3,4$, \\ Eppie Mildred Yiu, MBBS, PhD 2,3,4, R. John Massie, MBBS FRACP PhD 2,3,4, \\ Mark Domenic Pertile, PhD ${ }^{1,2,3}$, Desirée du Sart, PhD, FFSc(RCPA) $)^{1,2,3}$, \\ Damien Bruno, PhD, FFSc(RCPA $)^{1,2,3}$ and David. J. Amor, MBBS, PhD ${ }^{1,2,3,4}$
}

Purpose: To describe our experience of offering simultaneous genetic carrier screening for cystic fibrosis (CF), fragile $\mathrm{X}$ syndrome (FXS), and spinal muscular atrophy (SMA).

Methods: Carrier screening is offered through general practice, obstetrics, fertility, and genetics settings before or in early pregnancy. Carriers are offered genetic counseling with prenatal/ preimplantation genetic diagnosis available to those at increased risk.

Results: Screening of 12,000 individuals revealed 610 carriers (5.08\%; 1 in 20): 342 CF, 35 FXS, 241 SMA (8 carriers of 2 conditions), approximately $88 \%$ of whom had no family history. At least $94 \%$ of CF and SMA carriers' partners were tested. Fifty couples $(0.42 \% ; 1$ in 240$)$ were at increased risk of having a child with one of the conditions (14 CF, 35 FXS, and 1 SMA) with 32 pregnant at the time of testing. Of these, 26 opted for prenatal diagnosis revealing 7 pregnancies affected (4 CF, 2 FXS, 1 SMA).

Conclusion: The combined affected pregnancy rate is comparable to the population risk for Down syndrome, emphasizing the need to routinely offer carrier screening. The availability of appropriate genetic counseling support and a collaborative approach between laboratory teams, genetics services, health professionals offering screening, and support organizations is essential.

Genet Med advance online publication 26 October 2017

Key Words: carrier screening; cystic fibrosis; fragile $\mathrm{X}$ syndrome; prenatal diagnosis; spinal muscular atrophy

\section{INTRODUCTION}

Population genetic carrier screening provides individuals and couples with information about their risk of having a child with a genetic condition. Such programs were originally implemented for inherited conditions, one condition at a time; however, advances in genetic testing technology have enabled the development of "extended" carrier screening panels comprising multiple conditions. These panels test for multiple autosomalrecessive and $\mathrm{X}$-linked conditions, regardless of the presence or absence of family history, thereby informing reproductive decision making and planning.

There is debate about which conditions should be included in extended carrier screening panels. ${ }^{1}$ Criteria for inclusion of a genetic condition include that the condition should have a well-defined phenotype and a serious impact on the affected individual and their family, be relatively common, have a highly accurate test available with a high detection rate, be able to be diagnosed prenatally, and that screening for the condition is perceived to be acceptable to the target population and the community. ${ }^{2-4}$

Population-based carrier screening for cystic fibrosis has been available in Victoria, Australia since 2006., In late 2012, Victorian Clinical Genetics Services (VCGS) began offering a multidisorder genetic carrier screen, now offered under the brand name prepair ${ }^{\mathrm{TM}}$, comprising three common and severe conditions: cystic fibrosis (CF), spinal muscular atrophy (SMA), and fragile X syndrome (FXS) (Table 1). Here we report findings from the first 12,000 individuals screened.

\footnotetext{
${ }^{1}$ Victorian Clinical Genetics Services, Parkville, Victoria, Australia; ${ }^{2}$ Murdoch Childrens Research Institute, Parkville, Victoria, Australia; ${ }^{3}$ Department of Paediatrics, University of Melbourne, Parkville, Victoria, Australia; ${ }^{4}$ Royal Children’s Hospital, Parkville, Victoria, Australia. Correspondence: Melanie Smith (melanie.smith@vcgs.org.au) Alison Dalton Archibald and Melanie Jane Smith are co-first authors. 
Table 1 Characteristics of CF, FXS, and SMA and details about genetic testing

\begin{tabular}{|c|c|}
\hline Condition & Clinical features \\
\hline Cystic fibrosis & $\begin{array}{l}\text { Chronic suppurative lung disease, } \\
\text { pancreatic exocrine insufficiency, } \\
\text { blocked biliary ducts, elevated sweat } \\
\text { electrolytes, poor weight gain, and } \\
\text { infertility in males. }\end{array}$ \\
\hline
\end{tabular}

Mode of inheritance and cause Treatment/management of condition

Autosomal recessive.

Affected individuals have two

No cure. Advances in treatment have

led to increases in life expectancy

improvements in quality of life.

Greater than 2,000 disease-causing

CFTR variants have been identified.

Fragile $X$

syndrome

Developmental delay, intellectual

disability, speech delay, autistic-like behaviors, anxiety, ADHD, epilepsy, macrocephaly, large ears, long face. Features of FXS vary from mild to severe with males more likely to be severely affected than females.

\section{Spinal}

muscular

Progressive muscle weakness and

atrophy atrophy. Classified according to

maximal functional status achieved. Type 1: never sit unsupported, onset before 6 months, marked weakness and hypotonia, areflexia, tongue fasciculations, life expectancy $<2$ years from respiratory failure. Type

2 : sit independently but never stand or walk, onset between 6 and

18 months, proximal weakness, hand tremor, scoliosis, life expectancy $>2$ years to $3 r d / 4$ th decade. Type 3: stand and walk independently, onset after

18 months, may ultimately require wheelchair, life expectancy similar to normal population. ${ }^{b}$

\section{X-linked. Caused by the expansion}

of the CGG triplet repeat region of the FMR1 gene. FM $(200+C G G$ repeats) is associated with fragile $X$ syndrome. Female FM and PM carriers are at risk of having an affected child. Female PM carriers are at risk of fertility problems and early menopause and male and female carriers are at risk of fragile

$\mathrm{X}$-associated tremor/ataxia syndrome.

Autosomal recessive. SMA is due to Multidisciplinary management of homozygous deletions of the survival motor neuron gene (SMN1) remainder are compound heterozygotes for the deletion and an intragenic mutation of SMN1.

intervention, occupational and

efensiveness, hyperarousal, and

attention problems. Tailored

educational interventions.

Pharmacological treatments for

ADHD, anxiety, aggression, and under way for a number of drug therapies. pulmonary, gastrointestinal, modifying therapy for SMADecember 2016. in $95 \%$ of individuals. The
Prevalence and carrier frequency

1 in 2,500; 1 in 25 Most common lifethreatening recessive condition affecting

Purtralian children

romise in some cases.

speech therapy to address sensory mood instability. Clinical trials are nutritional, and orthopedic issues. The FDA approved the first diseasenusinersen-for all forms of SMA in

\section{1 in $10,000 / 1$ in 40 \\ Ascertaining SMN1 95\%}

SMA is the most

frequent genetic cause

of infant mortality. copy number.
Sizing of CGG repeat >99\% in $5^{\prime}$ region of FMR1

gene.

cause of inherited

intellectual disability.

Detection of

$90 \%^{a}$

variants in target

population. 


\section{MATERIALS AND METHODS}

\section{Screening program development}

The prepair ${ }^{\mathrm{TM}}$ (http://ww.vcgs.org.au/prepair) screening program was based on the CF carrier screening program offered by VCGS from 2005. 5,6 VCGS is a not-for-profit wholly owned subsidiary of the Murdoch Childrens Research Institute. Prepair ${ }^{\mathrm{TM}}$ is offered at AUD\$385 and is a privately funded test. Proceeds from testing are used by VCGS to improve program design and for medical research.

Partnerships were established with the local support organizations for CF, FXS, and SMA. The program was piloted in 2012 with 10 obstetricians offering screening, before being made available to general practitioners and obstetricians across Australia (primarily taken up in Victoria) in early 2013. Obstetricians, fertility specialists, general practitioners, genetic counselors, and clinical geneticists were notified of the screening program by letter and via newsletters and email bulletins. Education sessions were offered to all health professionals providing this test, and involved one-to-one or small group discussions and larger seminars. Information for patients and health professionals was available in an information brochure (see Supplementary Material online) and on the VCGS website.

\section{Screening program design}

Figure 1 summarizes the carrier screening process. Screening was offered to women prior to pregnancy or early in pregnancy (recommended $\leq 12$ weeks gestation) by general practitioners, obstetricians, fertility specialists, genetic counselors, and clinical geneticists. Screening for the three conditions was generally performed on the female first. Only $\mathrm{CF}$ and SMA carrier screening was performed when testing was requested for males. The purpose of this screening program was to identify individuals at risk of having a child with CF, FXS, and SMA. FXS carrier screening was not performed on males because male FMR1 premutation (PM) carriers are not at risk of having a child with FXS. Additionally, FXS PM carrier status conveys a risk for fragile X-associated tremor/ataxia syndrome, predominantly affecting males. It can therefore be argued that FXS carrier testing in males is predictive testing for fragile $\mathrm{X}$-associated tremor/ataxia syndrome requiring more comprehensive pretest genetic counseling, which is not feasible for a population screening program. ${ }^{7}$ An information brochure summarizing key information necessary for decision making was attached to the test request form and genetic counseling was available at any stage in the carrier screening process.

All carrier results were discussed with the referring health professional by telephone with genetic counseling offered for the carrier. For carriers of CF or SMA, genetic counseling was provided by telephone and testing of the partner was offered and arranged. All couples/individuals with an increased risk of the condition (CF and SMA carrier couples and FXS carrier females) were offered a genetic counseling appointment and an appointment with a pediatric subspecialist with expertise in the relevant condition.
To assess program outcomes, data were extracted from laboratory and genetic counseling databases. As the offer of testing was at the discretion of the individual health-care practitioner, we could not gather data about individuals who were offered but chose not to proceed with screening. Demographic and referral information were obtained from test request forms completed by the referring practitioner. As the genetic counseling team had contact with the carrier and/ or their referring practitioner, more detailed and comprehensive information was available for carriers.

\section{Genetic testing \\ Cystic fibrosis}

Testing was performed for 38 variants in the CFTR gene (Table 2), which account for $\sim 90 \%$ of CF carriers in the Australian Caucasian population. Inclusion of variants in the panel was based on the recommendations by the American College of Medical Genetics and Genomics (ACMG), their frequency in our population, and variant severity. Variants that result in mild and/or late-onset CF were not included following the recommendations of the Human Genetics Society of Australasia. ${ }^{8}$ Individuals with a known familial variant that was not included in the 38 panel were offered testing for that variant. Testing of the 38 panel was performed using matrix-assisted laser desorption ionization-time of flight mass spectrometry. Variants were detected by single-nucleotide primer extension analysis. If a variant failed testing, if a novel peak was observed in the mass spectrometry chromatogram, or if an individual had a known family variant, the specific region was analyzed by Sanger sequencing. If a new variant was identified, it was then assessed using previously published data to determine clinical significance. The variant was only reported if classified as pathogenic or likely pathogenic. If no CFTR variants were identified the result was reported as "low risk" of being a CF carrier.

\section{Fragile $X$ syndrome}

FXS carrier screening was performed by triplet-primed polymerase chain reaction of the FMR1 CGG repeat region using the FMR1 TP-PCR kit (Abbott, USA) followed by highthroughput automated capillary electrophoresis. This assay has a detection rate of $99 \%$, which enables detection of CGG repeat expansions and resolves normal homozygous female samples from heterozygotes with expansions. ${ }^{9}$ As this screening program was designed to identify individuals at increased risk of having a child with FXS, a threshold of 55 CGG repeats was chosen to differentiate normal from expanded alleles, as the lowest PM reported to have expanded to a full mutation (FM) is 56 CGG repeats. ${ }^{10}$ If a result showed $\leq 54$ repeats, it was reported as "low risk" for having a child with FXS. Gray zone (GZ) alleles (45-54 CGG repeats) were reported as "low risk" because women with GZ alleles are not at risk of having a child with FXS. Results of $\geq 55$ repeats were reported as "increased risk" for having a child with FXS. All samples from individuals with increased risk 


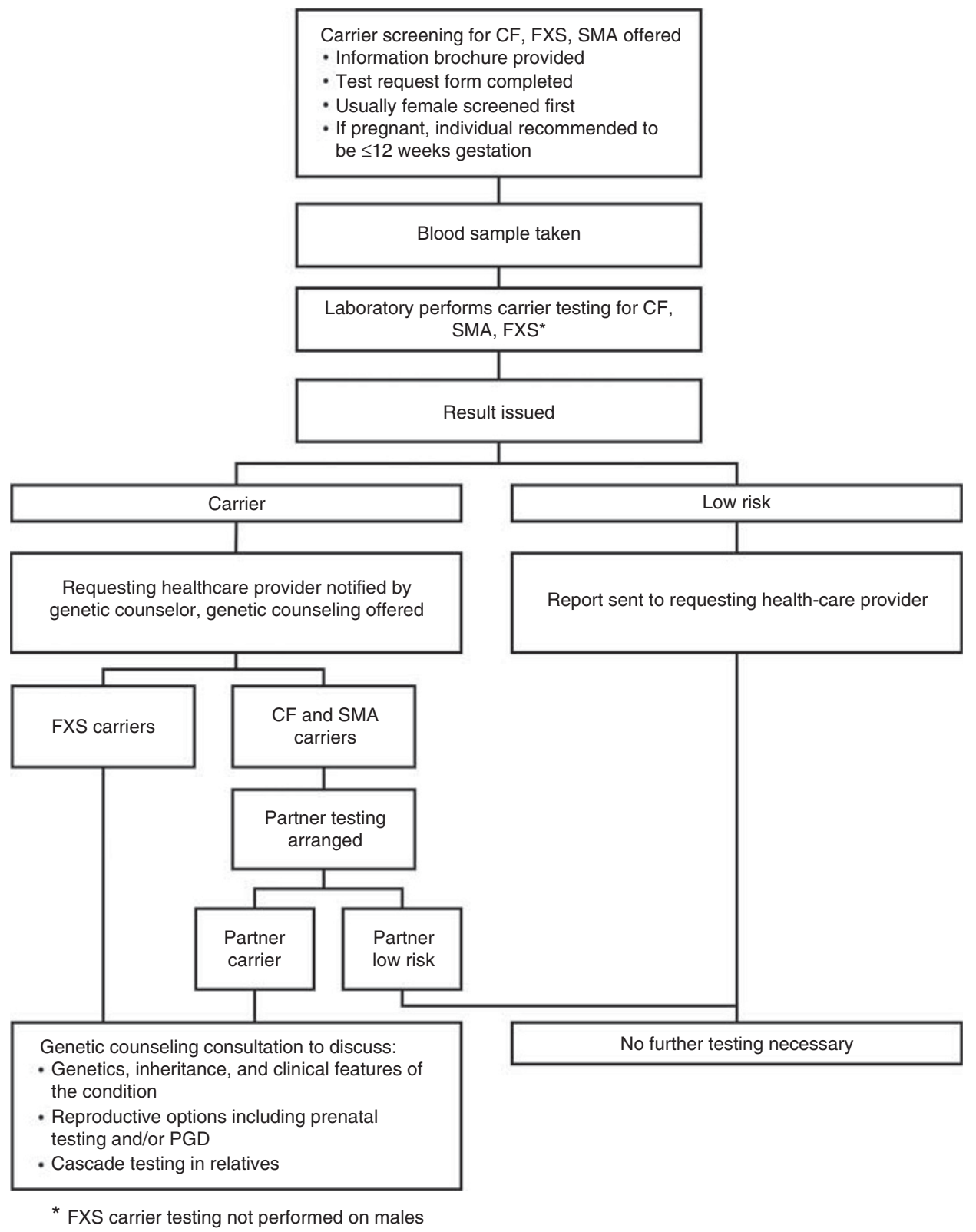

Figure 1 Carrier screening process. CF, cystic fibrosis; FXS, fragile $X$ syndrome; PGD, preimplantation genetic diagnosis; SMA, spinal muscular atrophy.

results had confirmatory testing through standard diagnostic testing protocols, ${ }^{11}$ including Southern blot analysis for individuals identified with an allele greater than 55 repeats. ${ }^{12}$ The results were always concordant within \pm 2 repeats.

\section{Spinal muscular atrophy}

A quantitative real-time polymerase chain reaction was used to measure the copy number of exon 7 of the SMN1 gene, as the vast majority of carriers have a deletion in exon 7 . This assay enables the specific copy number of the SMN1 gene to be determined. ${ }^{13}$ If two or more copies of the SMN1 gene were identified, the result was reported as "low risk" for being a carrier of SMA. If one copy of the SMN1 gene was identified, the result was reported as a "carrier" of SMA. This assay has a $95 \%$ sensitivity, as variants in untested regions of SMN1 as well as the $2+0$ genotype are not detected.

\section{Demographics}

Data from the first 12,000 individuals tested indicated that $96.92 \%(11,630)$ were female. The average age at carrier testing was 33.79 years (33.69 years for females and 36.86 years for males). Approximately $96 \%(11,510)$ of individuals screened were from Victoria, with $3.78 \%$ (453) from other Australian states or territories, and $0.15 \%$ (18) from overseas; for $0.16 \%$ (19) this information was not available. Of the 
Table 2 Allele frequencies for CF, FXS, and SMA

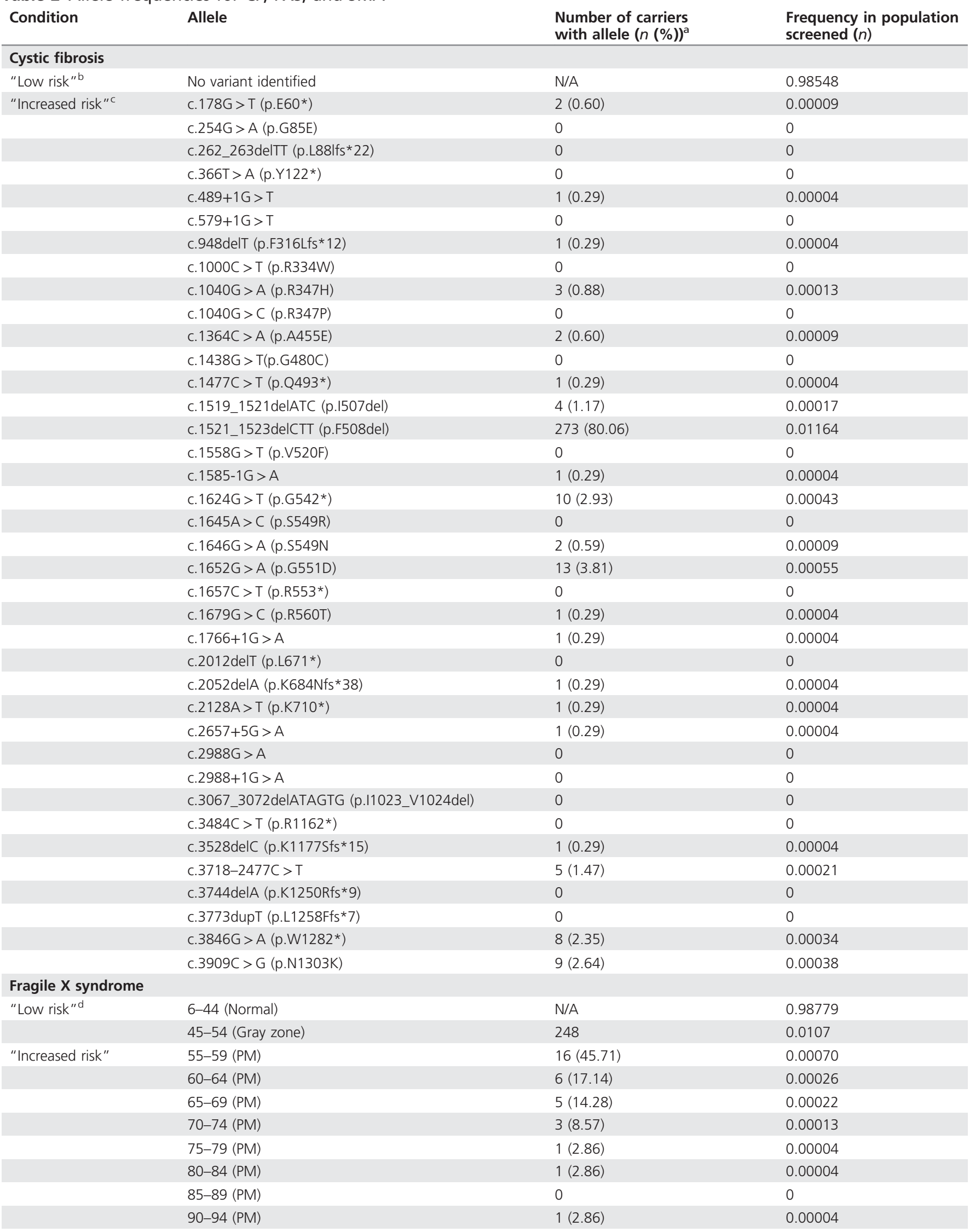


Table 2 Continued

\begin{tabular}{|c|c|c|c|}
\hline Condition & Allele & $\begin{array}{l}\text { Number of carriers } \\
\text { with allele }(n(\%))^{\text {a }}\end{array}$ & $\begin{array}{l}\text { Frequency in population } \\
\text { screened }(n)\end{array}$ \\
\hline & 95-99 (PM) & 0 & 0 \\
\hline & 100-199 (PM) & $1(2.86)$ & 0.00004 \\
\hline \multicolumn{4}{|c|}{ Spinal muscular atrophy } \\
\hline "Low risk" e & 2 Copies SMN1 & $10,907(90.99)$ & 0.90990 \\
\hline "Increased risk" ${ }^{f}$ & 1 copy SMN1 & $241(2.00)$ & 0.02000 \\
\hline
\end{tabular}

CF, cystic fibrosis; FM, full mutation; FXS, fragile X syndrome; PM, permutation; SMA, spinal muscular atrophy.

${ }^{\text {a }}$ For SMA, number of individuals with allele. ${ }^{\mathrm{b}}$ Estimated residual risk of CF for an individual with no variant identified and an untested partner is 1 in 24,100 . ${ }^{\mathrm{C}}$ Risk of $\mathrm{CF}$ for couples where both partners are CF carriers is 1 in 4, estimated residual risk of CF for an individual with an identified variant and partner with no variant identified is 1 in 964; 'No residual risk was assigned for individuals with a report issued as "low risk" for FXS due to it being an X-linked condition with a very high detection rate. eEstimated residual risk of SMA for an individual with no variant identified and an untested partner is 1 in 82,000 (2 copies SMN1), 1 in 248,000 (3 copies SMN1), 1 in 388,000 (4 copies SMN1). ${ }^{f}$ Risk of SMA for couples where both partners are SMA carriers is 1 in 4, estimated residual risk of SMA for an individual with an identified variant and partner with no variant identified is 1 in 2,035 (if partner has 2 copies of SMN1), 1 in 6,093 (if partner has 3 copies of SMN1), 1 in 9,478 (if partner has 4 copies of SMN1).

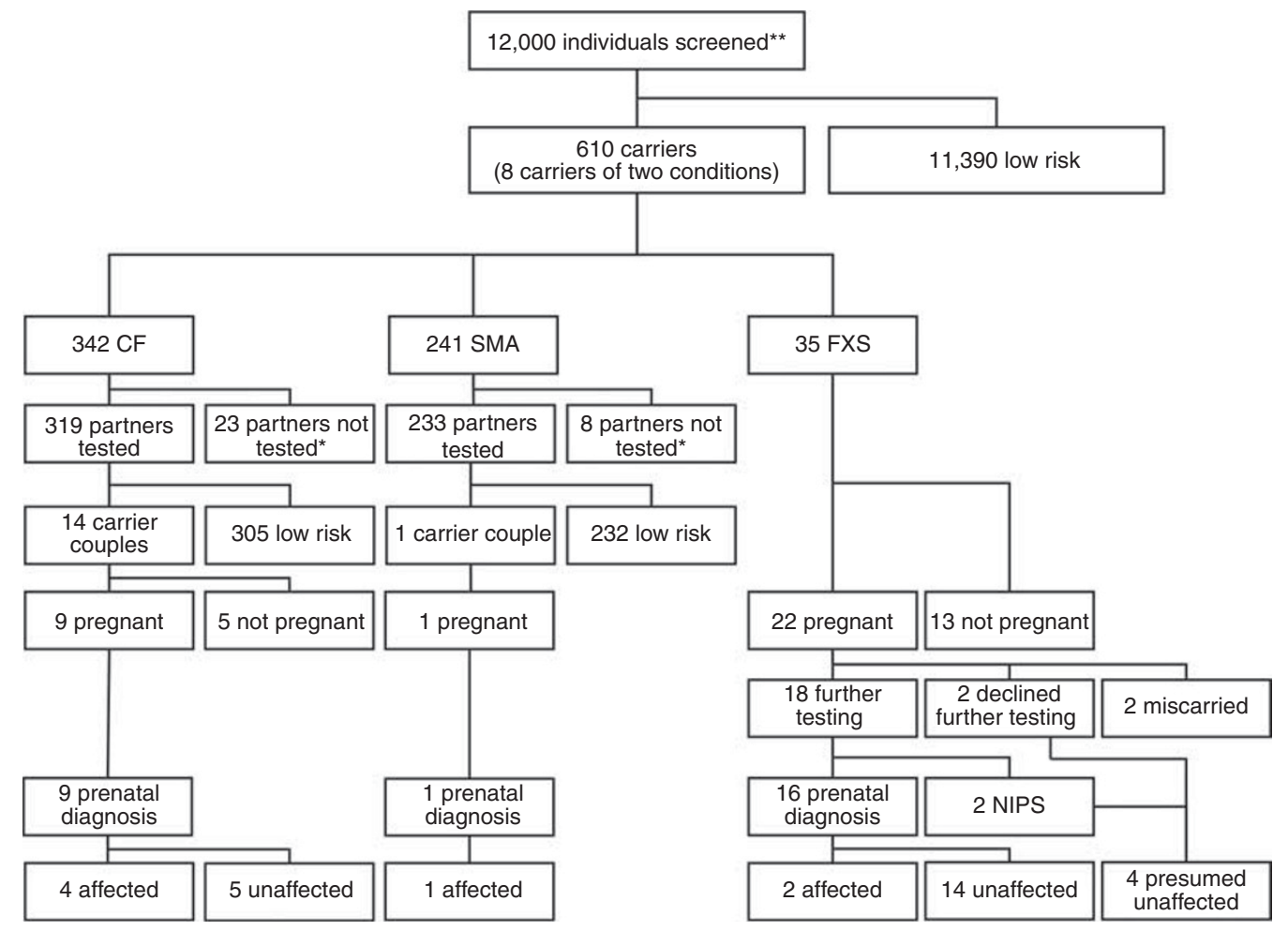

Figure 2 Outcomes of 12,000 individuals who had carrier screening for cystic fibrosis (CF), fragile X syndrome (FXS), and spinal muscular atrophy (SMA). NIPS, noninvasive prenatal screening. *Partners not tested" includes partners who declined testing or where it was not known whether the partner had been tested. ${ }^{*}$ In a small proportion of instances (CF 275, SMA 12), individuals were not tested for one of the conditions because they had previously had testing.

Victorian individuals screened, $95.80 \%(11,026)$ were from Greater Melbourne and 4.20\% (483) from regional Victoria. The proportions of tests ordered by health professional specialty were obstetricians $55.05 \%(6,606)$, fertility specialists $17.10 \%(2,052)$, doctors specializing as both obstetricians and fertility specialists $7.70 \%$ (924), general practitioners $15.83 \%$ $(1,900)$, clinical geneticists and genetic counselors $4.18 \%$ (502), and "other" $0.13 \%$ (16). Information provided on the request forms indicated that at least $69 \%$ of the female individuals screened were pregnant when tested. The majority 
of results $(93.63 \%)$ were reported within 10 working days from sample receipt, and $99.70 \%$ within 15 working days.

\section{Carriers}

Of 12,000 individuals screened, 610 ( 1 in $20 ; 5.08 \%$ ) were found to be carriers of at least one of the three conditions: 342 CF (1 in $34 ; 2.91 \%)$, 35 FXS ( 1 in $330 ; 0.30 \%$ ), and 241 SMA (1 in $50 ; 2.01 \%$ ) (Figure 2). Eight of these 610 individuals were carriers of two conditions (5 CF and SMA, 1 SMA and FXS, $2 \mathrm{CF}$ and FXS). Allele frequencies of the variants identified are summarized in Table 2. Of 22 CFTR variants that were detected in 342 CF carriers, 331 (96.78\%) were carriers of variants recommended for screening by ACMG, 10 $(2.92 \%)$ were carriers of six variants that are included in our customized panel, and one $(0.29 \%)$ was a carrier of a familyspecific variant.

At least $94.68 \%(552 / 583)$ of partners of CF and SMA carriers were tested to determine the couple's risk of having a child with the condition. In 20 cases we could not establish whether the partner had been tested and in 11 cases partner testing was not undertaken. Reasons for partner testing not being undertaken included the following: the person receiving the carrier result did not have a partner (6), the partner declined testing (2), the partner intends to have carrier testing but has not yet done so (1), the couple felt they would not terminate an affected pregnancy (1), and the partner of a CF carrier was of non-Caucasian ancestry and perceived themselves to be at low risk of being a carrier (1).

Prior to testing, $88.36 \%$ of carriers (539/610) had no known family history of the condition. The majority of carriers were pregnant at the time of testing $(58.69 \%, 358 / 610)$.

\section{Outcomes from increased risk individuals/couples}

Fifty individuals/couples $(0.42 \%$ or 1 in 240 people screened) were at increased risk of having a child with one of the three conditions (14 CF, $35 \mathrm{FXS}$, and 1 SMA). Of these, a family history of the condition was present for 13 (26\%): six CF carrier couples and seven FXS carriers. For 12 of the $14 \mathrm{CF}$ carrier couples, both partners were carriers of a CFTR variant in the ACMG list of 23 variants recommended for population screening. ${ }^{14}$ In one instance, one member of the couple carried a variant not in the ACMG list, and in another, the variant information of the partner of one of the carriers was not provided (partner had been tested by another laboratory). Most (24/35; 68.50\%) FXS carriers had a small PM of 65 CGG repeats or less conveying a risk of expansion to FM of less than $5 \%$. $^{15}$

As outlined in Figure 2, 64\% (32) of the increased risk couples were pregnant at the time of testing (9 CF, 22 FXS, 1 SMA). All CF and SMA carrier couples opted for prenatal diagnosis, as did 16 of the FXS carriers, and seven affected pregnancies were identified (4 CF, 2 FXS, 1 SMA). After prenatal SMN1 testing revealed 0 copies, SMN2 copy-number prenatal testing was performed on the affected fetus. This showed two copies of SMN2 in the fetus, indicating a high risk for an early-onset form of SMA. ${ }^{16}$ This couple chose termination of pregnancy, as did three of the four couples with CF-affected pregnancies.

Of the 16 FXS PM carriers who had prenatal diagnosis, the expanded FMR1 allele was inherited by the fetus in 10 cases. Of these, two FMR1 alleles expanded further but remained in the PM range $(56 \rightarrow 62 ; 67 \rightarrow 78)$, and two PMs expanded to FMs $\quad(71 \rightarrow 113 / 1,607$ mosaic male; $123 \rightarrow 508-1,118$ female). Both couples with FM CVS results chose termination of pregnancy. Of the six PM carriers who did not have prenatal diagnosis, two miscarried, and four declined prenatal diagnosis: one (55 CGG repeats) declined prenatal diagnosis due to the low risk of FXS, two (55 and 57 CGG repeats) used noninvasive prenatal screening to determine the sex of the fetus and opted not to undergo prenatal diagnosis after discovering the fetus was female, and one (72 CGG repeats) did not have further testing as she would not terminate an affected pregnancy.

\section{Additional findings}

Additional findings were identified in six individuals. FXS testing identified one woman who likely had 47,XXX, as three FMR1 CGG repeat lengths were identified (23, 29, and 30 CGG repeats); this result was reported as "low risk." Five individuals were identified with CFTR variants that were not part of the 38-variant panel (Table 3 ). This arose because the matrix-assisted laser desorption ionization-time of flight spectrometry system can detect novel alleles as aberrant peaks or reduced peaks on the mass spectrometry chromatograms that are then confirmed by sequencing analysis. Four of the CF variants (c.374T $>$ C, c.1438G $>$ A, c. $1666 \mathrm{~A}>\mathrm{G}$, c.3854C $>\mathrm{T}$ ) had been reported in the Cystic Fibrosis Mutation Database (http://www.genet.sickkids.on.ca/cftr/ app) in patients with milder CFTR-related presentations, including congenital bilateral absence of the vas deferens and CF-like symptoms. These variants were reported as "low risk." Information obtained from in silico software predicted the pathogenicity of the fifth $\mathrm{CF}$ variant $(\mathrm{c} .1364 \mathrm{C}>\mathrm{T})$, to be disease-causing, but due to the absence of reported clinical cases, this variant was classified as a variant of unknown significance (VUS) and reported as "low risk."

\section{DISCUSSION}

We have demonstrated high clinical utility in population screening for CF, FXS, and SMA, three inherited conditions for which there is a strong rationale for population carrier screening. Our program was the first genetic carrier screening panel available in Australia offering simultaneous screening for these conditions. With significant interest from health professionals and those offered screening, we have shown that a comprehensive screening program can be offered successfully through a specialist genetics service with strong clinical and laboratory expertise. This has enabled many individuals to become aware of their carrier status and to obtain an accurate assessment of their risk of having a child with these conditions. 


\begin{abstract}
Table 3 CF variants identified in five individuals who were not part of the 38 CFTR variant panel Variant Classification

c. 374 T >C A heterozygous missense variant was identified NM_000492(CFTR):C.374T >C in exon 4 of the CFTR gene. This substitution is predicted to create a change of an isoleucine to a threonine at amino acid position 125, NP_000483.3(CFTR):p.(Ile125Thr). The amino acid at this position is highly conserved and is not situated in a known functional domain. The Grantham assessment of amino acid properties and conservation indicates that this change is equivocal. In silico software predictions of the pathogenicity of this variant are conflicting. This variant has not been previously observed in our patient cohort, but is listed on the Cystic Fibrosis Mutation Database and no phenotypic information is available. It is cited in several publications as a variant associated with CF and CF-like symptoms in Asian populations. It is present in the ExAC database at a frequency of less than $1 \%$ and 1 homozygote has been reported in the East Asian population. Based on the current information, this variant has been classified as a VUS.

C.1364C > T A heterozygous missense variant was identified NM_000492(CFTR):C.1364C > T in exon 10 of the CFTR gene. This substitution is predicted to create a change of an alanine to a valine at amino acid position 455, NP_000483.3(CFTR):p.(Ala455Val). The amino acid at this position is highly conserved and is not situated in a known functional domain. The Grantham assessment of amino acid properties and conservation indicates that this change is deleterious. In silico software predicts this variant to be disease-causing. This variant has not been previously observed in our patient cohort, and has not been previously reported in other clinical cases. Based on current information, this variant has been classified as a VUS.

c.1438G > A A heterozygous missense variant was identified NM_000492(CFTR):c.1438G > A in exon 11 of the CFTR gene. This substitution is predicted to create a change of a glycine to a serine at amino acid position 480, NP_000483.3(CFTR):p.(Gly480Ser). The amino acid at this position is highly conserved and is not situated in a known functional domain. The Grantham assessment of amino acid properties and conservation indicates that this change is deleterious. In silico software predicts this variant to be disease causing. This variant has not been previously observed in our patient cohort, but is listed on the Cystic Fibrosis Mutation Database and in a CBAVD patient. Based on current information, this variant has been classified as a VUS.

c.1666A > G A homozygous missense variant was identified NM_000492(CFTR):C.1666A > G in exon 12 of the CFTR gene. This substitution is predicted to create a change of an isoleucine to a valine at amino acid position 556, NP_000483.3(CFTR):p.(Ile556Val). The amino acid at this position is moderately conserved and is situated in the NBF1 domain. The Grantham assessment of amino acid properties and conservation indicates that this change is unlikely deleterious. In silico software predictions of the pathogenicity of this variant are conflicting. This variant has not been previously observed in our patient cohort, but is listed in the Cystic Fibrosis Mutation Database and in one additional patient with CBAVD. The variant is present in the ExAC database with 12 homozygote individuals in the East Asian population. Based on current information, this variant has been classified as a VUS.

c.3854C > T A homozygous missense variant was identified NM_000492(CFTR):C.3854C > T in exon 23 of the CFTR gene. This substitution is predicted to create a change of an alanine to a valine at amino acid position 1285, NP_000483.3(CFTR):p.(Ala1285Val). In ClinVar the clinical significance is not provided. This variant has not been previously observed in our patient cohort, but is listed in the Cystic Fibrosis Mutation Database in a CBAVD patient (Asian/Indian patient). In the ExAC database it is present at 0.5\% frequency in South Asians. Based on current information, this variant has been classified as a VUS.
\end{abstract}

CBAVD, congenital bilateral absence of the vas deferens; CF, cystic fibrosis; VUS, variant of unknown significance.

Our program revealed approximately 1 in 20 individuals to be a carrier of one or more of the conditions tested, and for the majority there was no known family history of the condition. One in 240 couples was identified as having a high chance of having an affected child. One in 1,006 women who were identified as carriers and pregnant at the time of testing had an affected pregnancy (4 CF, 2 FXS, 1 SMA). This affected pregnancy rate is comparable to the frequency of live births affected by Down syndrome, ${ }^{17}$ for which screening is routinely offered as part of standard pregnancy care. ${ }^{18}$ The high proportion of individuals identified as carriers emphasizes the benefits of implementing a population-based carrier screening approach rather than relying on family history to guide screening decisions.

Screening a large number of individuals has provided a useful insight into the carrier frequency in the local population with the proportion of individuals identified as carriers of CF and SMA (1 in 34 and 1 in 50 respectively) confirming previous reports in the Victorian population (CF:
1 in 33 (ref. 5); SMA: 1 in 49 (ref. 13)). Although there are limited Australian data available on FXS carrier frequency, 1 in 330 females screened were carriers ( $>54$ repeats) which is lower than that observed in North American populations ${ }^{19}$ and may be due to sample bias.

The provision of genetic counseling services and its coordination with laboratory testing forms a key component of the carrier screening program. This approach enabled appropriate management of additional findings of unclassified CFTR variants and possible triple $\mathrm{X}$ syndrome, and resulted in approximately $95 \%$ of partners of CF or SMA carriers being tested. This allowed accurate residual risk calculations for individuals with low risk results (based on carrier rates in the Australian Caucasian population) and for carriers with a partner who had a low risk result. The availability of genetic counseling, including the option of an appointment with a pediatric subspecialist, was valued by carriers and is fundamental given that informed decision making is difficult to achieve in the context of population screening. ${ }^{20,21}$ Since 
the advent of technologies like massively paralleled sequencing there have been a number of tests developed that screen for an expanded group of recessive disorders. There are estimated to be greater than 1,300 known recessive genetic conditions, affecting at least 30 in every 10,000 children, ${ }^{22}$ which collectively are anticipated to result in 1-2 in 100 couples being at high risk of having an affected child. To deliver screening programs with high clinical utility, careful consideration needs to be given to carrier screening program design before implementation, including the conditions to be screened, the detection rate for each condition, the current knowledge base for known variants, and ethnic difference in carrier frequencies. ${ }^{2,4}$

Complex inheritance patterns and disease etiology of $\mathrm{CF}$, SMA, and FXS raised interesting genetic counseling considerations. There are approximately 2,000 gene sequence variants that have been identified in the CFTR gene ${ }^{23}$ with only 23 of the most common disease-causing variants recommended for testing by ACMG based on known pathogenicity and population frequency. The considerable variation of carrier frequencies and pathogenic variants between different ethnic groups is a key challenge in developing population screening programs for CF. Ethnic variation was highlighted in our program with some individuals identified as carriers of CFTR variants not in the ACMG list of recommended CFTR variants to screen for, including one member of a carrier couple who had an affected pregnancy. This emphasizes the need for population screening programs to be developed with the ethnic diversity of the local population in mind. We recognize that a limitation with our program, which is offered in a very diverse ethnic population, is the level of knowledge about sequence variants in some ethnic groups.

FXS carrier screening provided a number of genetic counseling complexities. Firstly, we opted to report results for women with GZ alleles (allele sizes between 45 and 54 CGG repeats) as "low risk" given that women with a GZ allele are not at risk of having a child with FXS. Our data indicate that approximately $2.13 \%$ of women tested had GZ alleles that, if not reported as "low risk," would have significantly impacted on genetic counseling resources and may have led to increased anxiety for these women and to increased uptake of invasive testing (CVS or amniocentesis). Secondly, a substantial proportion of women with PM alleles had PMs less than 65 CGG repeats, conveying a less than $5 \%$ chance of having a child with FXS. ${ }^{15}$ The genetic counseling for these women was challenging given that they generally had no family history of FMR1-associated disorders and the CGG was unlikely to expand to a FM in one generation. Most of the carriers of small PMs who were pregnant at the time of testing opted for prenatal diagnosis revealing stable transmissions when the premutation was passed on in all but one instance (where the PM increased by four repeats). It could be argued that these invasive diagnostic procedures may have been unnecessary. ${ }^{24}$ Presence of AGG interruptions influences stability of the FMR1 CGG repeat, ${ }^{25}$ and AGG analysis may provide more accurate estimates of the risk of expansion, assisting women with small PMs in making informed decisions, particularly regarding whether to proceed with invasive testing. ${ }^{26}$ Thirdly, offering FXS carriers the option of noninvasive prenatal screening to determine fetal sex followed by prenatal diagnosis for a male fetus, which in both cases was suggested by the patient's obstetrician, may reflect a lack of awareness that females can be affected by FXS, suggesting that more education about FXS for health professionals offering carrier screening is needed.

The wide spectrum of phenotypic severity seen in SMA posed interesting genetic counseling challenges in the case of SMA prenatal diagnosis. SMN2 copy-number testing was performed to guide counseling about the predicted phenotype of the affected fetus. An important genetic modifier of SMA and differing from SMN1 by only five nucleotides, SMN2 copy number is generally inversely correlated with severity. In an individual with SMA and two copies of SMN2, the probability of SMA types 1, 2, and 3 is $97.30 \%, 2.70 \%$, and $0.04 \%$ respectively. ${ }^{16}$ As the affected fetus had two copies of SMN2, it was highly likely to have an early-onset form of SMA (i.e., SMA type 1 or 2). However, as other genetic modifiers and factors affecting SMA severity exist, SMA type is difficult to predict with certainty and SMN2 copy number should be interpreted with caution in the context of prenatal testing. ${ }^{27}$ At the time of testing, clinical trials for infants with SMA were available, adding to the complexity of the decision making and genetic counseling for this couple. The recent FDA approval of nusinersen for all forms of $\mathrm{SMA},{ }^{28}$ and the importance of early treatment to maximize therapeutic benefit, ${ }^{16}$ reinforces the need for early and/or prenatal diagnosis of affected infants with SMA.

Our screening program is limited by the fact that it was predominantly offered by private practitioners (obstetricians and fertility specialists) in metropolitan Melbourne. This may have been due to a lack of knowledge and awareness of the availability of the test amongst general practitioners and health professionals in the public system. Additionally, a higher proportion of people screened were from Greater Melbourne (95.80\%) compared with the general Victorian population for which residents of Greater Melbourne make up $76.50 \%$ and account for $78.40 \%$ of births. ${ }^{29}$ The reasons for this are unclear but may relate to awareness and availability of the test in regional areas. Residents of regional Victoria have similar median income and a slightly lower proportion have a post-school academic qualification. ${ }^{29}$ The cost of the test may also have been a barrier. Information on the test request form provided by the health-care practitioner was extremely limited in some instances, and is the reason we have only been able to report presence or absence of family history for the carriers identified. We also did not collect information about the ancestral heritage of individuals screened, but this is likely to be similar to the general Victorian population in which the most frequently reported ancestries are Great Britain (80\%), Continental Europe (23\%), and Asia (13\%). ${ }^{29}$ We were not able to obtain a complete data set of pregnancy 
outcomes of increased risk couples/individuals for subsequent pregnancies, or for increased risk couples who were not pregnant at the time of learning their carrier status, due to people being seen at other genetics services or in vitro fertilization clinics, and/or moving interstate or overseas. Additionally, we were not able to obtain information on how many people were offered screening or why they may have declined this offer. However, research into our prior CF carrier screening program has shown that reasons for declining CF carrier screening include perceiving carrier screening as irrelevant due to a lack of family history of CF or other genetic conditions, and not intending to consider termination of pregnancy for CF. ${ }^{30}$ Health professional attendance at the screening program education sessions was unable to be recorded in some instances so we do not know the proportion of health professionals offering the test who attended an education session. Further research is needed to explore approaches to offering carrier screening panels, including the knowledge and perspectives of those offering and being offered this screening.

This genetic carrier screening program for CF, FXS, and SMA has proven to be successful in identifying individuals and couples at increased risk of having a child affected by one of these conditions. Offering screening through a coordinated clinical and laboratory service ensures patients are supported to make informed reproductive choices. This program has demonstrated that despite individual recessive conditions being relatively rare, when tested collectively, the combined chance of an affected child with one of the conditions is comparable to that of Down syndrome. This screening program establishes a solid foundation and working protocol for expansion to include a larger number of genetic conditions.

\section{SUPPLEMENTARY MATERIAL}

Supplementary material is linked to the online version of the paper at http://www.nature.com/gim

\section{ACKNOWLEDGMENTS}

We acknowledge Cystic Fibrosis Victoria, Fragile $X$ Alliance (Jonathan Cohen), Fragile $X$ Association of Australia, and Spinal Muscular Atrophy Association of Australia (Julie Cini) for their encouragement and input into the development of this screening program. We thank the members of the original CF carrier screening program team (Martin Delatycki, Agnes Bankier, Lisette Curnow, Robin Forbes, Vicki Petrou, Liane loannou, Desirée du Sart, Steven Nasioulas, Melanie Smith, and John Massie). We also acknowledge the contribution of Deborah Dalton, Associate Genetic Counselor.

\section{DISCLOSURE}

The authors declare no conflict of interest.

\section{REFERENCES}

1. Henneman L, Borry P, Chokoshvili $D$, et al. Responsible implementation of expanded carrier screening. Eur J Hum Genet. 2016;24:e1-e12.

2. Committee opinion no. 690 summary: carrier screening in the age of genomic medicine. Obstet Gynecol. 2017;129:595-596.
3. Edwards JG, Feldman G, Goldberg J, et al. Expanded carrier screening in reproductive medicine-points to consider: a joint statement of the American College of Medical Genetics and Genomics, American College of Obstetricians and Gynecologists, National Society of Genetic Counselors, Perinatal Quality Foundation, and Society for MaternalFetal Medicine. Obstet Gynecol. 2015;125:653-662.

4. Grody WW, Thompson BH, Gregg AR, et al. ACMG position statement on prenatal/preconception expanded carrier screening. Genet Med. 2013;15:482-483.

5. Archibald AD, Massie J, Smith MJ, Dalton DG, du Sart D \& Amor DJ. Population-based genetic carrier screening for cystic fibrosis in Victoria. Med J Aust. 2014;200:205-6

6. Massie J, Petrou V, Forbes R, et al. Population-based carrier screening for cystic fibrosis in Victoria: the first three years experience. Aust N Z J Obstet Gynaecol. 2009;49:484-489.

7. Archibald $A D$, Hickerton $C L$, Jaques $A M$, Wake S, Cohen J \& Metcalfe SA. "It's about having the choice": stakeholder perceptions of populationbased genetic carrier screening for fragile X syndrome. Am J Med Genet A. 2013;161:48-58.

8. Delatycki MB, Burke J, Christie L, et al. Human Genetics Society of Australasia position statement: population-based carrier screening for cystic fibrosis. Twin Res Hum Genet. 2014;17:578-583.

9. Tassone F, Pan R, Amiri K, Taylor AK \& Hagerman PJ. A rapid polymerase chain reaction-based screening method for identification of all expanded alleles of the fragile X (FMR1) gene in newborn and high-risk populations. J Mol Diagn. 2008;10:43-49.

10. Fernandez-Carvajal I, Lopez Posadas B, Pan R, Raske C, Hagerman PJ \& Tassone F. Expansion of an FMR1 grey-zone allele to a full mutation in two generations. J Mol Diagn. 2009;11:306-310.

11. Khaniani MS, Kalitsis P, Burgess T \& Slater HR. An improved diagnostic PCR assay for identification of cryptic heterozygosity for CGG triplet repeat alleles in the fragile X gene (FMR1). Mol Cytogenet. 2008;1:5.

12. Yu S, Pritchard $M$, Kremer $E$, et al. Fragile $X$ genotype characterized by an unstable region of DNA. Science. 1991;252:1179-1181.

13. Smith M, Calabro V, Chong B, Gardiner N, Cowie S \& du Sart D. Population screening and cascade testing for carriers of SMA. Eur J Hum Genet. 2007;15:759-766.

14. Grody WW, Cutting GR, Klinger KW, Richards CS, Watson MS \& Desnick RJ. Laboratory standards and guidelines for population-based cystic fibrosis carrier screening. Genet Med. 2001;3:149-154.

15. Nolin SL, Brown WT, Glicksman A, et al. Expansion of the fragile X CGG repeat in females with premutation or intermediate alleles. Am J Hum Genet. 2003;72:454-464

16. Burns JK, Kothary R \& Parks RJ. Opening the window: the case for carrier and perinatal screening for spinal muscular atrophy. Neuromuscul Disord. 2016;26:551-559

17. Hook EB, Cross PK \& Schreinemachers DM. Chromosomal abnormality rates at amniocentesis and in live-born infants. JAMA. 1983;249: 2034-2038.

18. Prenatal screening and diagnosis of chromosomal and genetic abnormalities in the fetus in pregnancy. RANZCOG statement C-Obs59. March 2015. https://www.ranzcog.edu.au.

19. Seltzer MM, Baker MW, Hong J, Maenner M, Greenberg J \& Mandel D. Prevalence of CGG Expansions of the FMR1 Gene in a US PopulationBased Sample. Am J Med Genet B Neuropsychiatr Genet. 2012;159: 589-597.

20. Archibald $A D$, Hickerton $C L$, Wake $S A$, Jaques $A M$, Cohen J \& Metcalfe SA. "It gives them more options": preferences for preconception genetic carrier screening for fragile $X$ syndrome in primary healthcare. J Community Genet. 2016;7:159-171.

21. Beard CA, Amor DJ, Di Pietro L \& Archibald AD. "I'm healthy, it's not going to be me": exploring experiences of carriers identified through a population reproductive genetic carrier screening panel in Australia. Am J Med Genet A. 2016;170:2052-2059.

22. Henneman L, Borry P, Chokoshvili D, et al. Responsible implementation of expanded carrier screening. Eur J Hum Genet. 2016:24:e1-e12.

23. Gallati S. Disease-modifying genes and monogenic disorders: experience in cystic fibrosis. App/ Clin Genet. 2014;7:133-146.

24. Grody WW. Where to draw the boundaries for prenatal carrier screening. JAMA. 2016;316:717-719.

25. Yrigollen CM, Martorell L, Durbin-Johnson B, et al. AGG interruptions and maternal age affect FMR1 CGG repeat allele stability during transmission. J Neurodev Disord. 2014;6:24. 
26. Finucane $B$, Lincoln S, Bailey L \& Martin CL. Prognostic dilemmas and genetic counseling for prenatally detected fragile $X$ gene expansions. Prenat Diagn. 2017;37:37-42.

27. Prior TW, Nagan N, Sugarman EA, Batish SD \& Braastad C. Technical standards and guidelines for spinal muscular atrophy testing. Genet Med. 2011;13:686-694.

28. US Food and Drug Administration. FDA approves first drug for spinal muscular atrophy. 2016. https://www.fda.gov/newsevents/ newsroom/pressannouncements/ucm534611.htm. Accessed 20 June 2017.

29. Australian Bureau of Statistics Quick Stats. 2016. https://www.abs.gov. au. Accessed 1 June 2017.

30. Ioannou L, Massie J, Lewis S, McClaren B, Collins V \& Delatycki MB. 'No thanks'-reasons why pregnant women declined an offer of cystic fibrosis carrier screening. J Community Genet. 2014;5:109-117. 